\title{
Positive placement experience and future rural practice intentions: findings from a repeated cross-sectional study
}

This article was published in the following Dove Press journal: Journal of Multidisciplinary Healthcare

\author{
Yaqoot Fatima' \\ Sarvat Kazmi \\ Stephanie King' \\ Shaun Solomon' \\ Sabina Knight' \\ 'Centre for Rural and Remote Health \\ (Mount Isa), James Cook University, \\ Mount Isa, QLD, Australia; ${ }^{2}$ School of \\ Health Science, Massey University, \\ Wellington, New Zealand
}

Objective: To identify the constituents of positive placement experience and explore the association between positive placement experiences and rural and remote practice intentions.

Methods: A repeated cross-sectional study was conducted between January 2014 and December 2017. Medical, nursing, dentistry, and allied health students who completed a rural/remote placement were invited to complete a survey questionnaire on placement experience. Information on students' sociodemographic factors, discipline, placement experience, placement satisfaction, and rural/remote practice intentions was collected. Modified Poisson regression was performed to determine the relationships between placement satisfaction and future rural practice intentions. Qualitative data were analyzed by thematic analysis.

Results : A total of 873 students responded. The majority of the survey respondents were females $(70.06 \%$ ), with a median age of 22 years (IQR $21-25$ years), and $44.46 \%$ of respondents were medical students. Students satisfied with their placement were 2.10 times more likely to have rural/remote practice intention than their counterparts. Of all components of rural/remote placement experience, satisfaction with the placement supervision had the highest impact on changing students' rural/remote practice intentions from negative to positive. The major themes from qualitative analysis were as follows: "wide variety of experience and hands-on learning opportunities," "multidisciplinary exposure at home and workplace," "support from the local University Department of Rural Health (UDRH)," "learning of indigenous culture," and "experiencing challenges of rural health care services."

Conclusion: There is a strong association between positive placement experience and future rural/remote practice intentions. Therefore, facilitation of positive placement experiences in remote and rural locations could be a key strategy in addressing rural health workforce maldistribution.

Keywords: rural health workforce, student placement, postplacement practice intentions

\section{Introduction}

Maldistribution of the health workforce in rural and remote locations has led to initiatives encouraging clinicians going for rural and remote practice. ${ }^{1}$ Empirical evidence demonstrates that rural background, scholarships, and financial incentives to undertake a rural placement are associated with future rural practice intentions., ${ }^{2,3}$ While rural origin is a strong predictor of future rural practice, not all rural students practice at their place of origin, as only $<20 \%$ rural students are reported to be practicing rurally. ${ }^{4}$ Therefore, while persistent efforts are required to retain rural students for future local practice, more effective strategies are needed to encourage and involve urban health care providers for rural practice.
Correspondence: Yaqoot Fatima Centre for Rural and Remote Health (Mount Isa), James Cook University, Mount Isa, QLD 4825, Australia Tel +6I 0747454529

Email yaqoot.fatima@jcu.edu.au 
Rural clinical placements are an ideal platform to provide "rural exposure" to students by providing a snapshot of what it would be like practicing and living in the rural community. ${ }^{5}$ Some studies have shown that extended clinical placement in rural locations help students in immersing themselves in the local community and provide a more realistic experience of living and working as a rural practitioner. ${ }^{6,7}$

It is also important to consider that not all rural placements are of comparable duration for students from different health care disciplines. While medicine and allied health students have more extended placement (16-20 weeks), nursing and pharmacy students stay for a short duration (2-8 weeks). Consequently, their exposure and immersion in the community also vary. This variation in immersion in rural communities significantly affects their opportunity for community exposure as the experience is abridged. They also get lesser opportunity to experience the interprofessional activities due to time constraints.

Due to the lack of information on confounding variables, evidence for the role of placement experience in promoting future rural practice is inconclusive. ${ }^{8,9}$ Nonetheless, preliminary evidence suggests that positive placement experience significantly influences students' decision to continue remote practice. ${ }^{10}$ Therefore, exploring the elements constituting positive placement experience and subsequent improvement of rural placements could be a potential intervention to tackle maldistribution of the rural health workforce. Unfortunately, most of the work on the role of rural/remote placement experience on future rural/remote practice intentions has been mainly done for medicine students, ${ }^{11,12}$ with only a few studies exploring this link for other health care disciplines. ${ }^{2,13}$

While it is crucial to explore specific aspects of rural/ remote clinical placement that contribute to the positive experience, it is also crucial to identify placement elements that have the highest impact on future rural/remote practice intentions so that appropriate strategies could be used to meet students' expectations. This study aims to explore health care students' rural/remote placement experiences, identify the elements that are significantly linked with future rural/remote practice intentions, and assess if satisfaction with placement experience can change students' preplacement "negative" rural/remote practice intentions into "positive" intentions.

\section{Methods}

\section{Study setting and participants}

Mount Isa Centre for Rural and Remote Health (MICRRH) is one of the national network of 11 University Department of Rural Health (UDRH) center, providing placement opportunities for undergraduate, postgraduate, and international students. MICRRH provides academic support to students, as well as travel and accommodation subsidies, information technology access, and pastoral support while they are on placement. To facilitate the organization of placements, MICRRH has a full-time dedicated placement officer as the central point of contact for all students placed at MICRRH. The placement officer conducts the mandatory orientation program for new students upon their arrival. All students who attend MICRRH attend 1-day cultural awareness course during their placement. This orientation program helps the students understand the local approach to indigenous health care delivery throughout the area serviced by MICRRH. Toward the end of their placement, students are invited to complete an online evaluation survey that contains all the 21 core questions that are asked by all UDRHs.

\section{Study design}

The present study is a repeated cross-sectional study, involving students who undertook a clinical placement at a rural and remote location in North West Queensland, Australia, and were supported by the local UDRH (MICRRH). All placement students were invited to participate in this survey. The survey questionnaire was repeatedly administered in 2014, 2015, 2016, and 2017. Although survey participation was voluntary, students were actively encouraged to participate in the survey. Survey participants were informed of the purpose of the survey and consented to participation.

\section{Ethics approval}

The ethics application was approved by the James Cook University Human Research Ethics Committee (H6899). Deidentified data from the study were used for analysis.

\section{Survey instruments}

The questionnaire was developed collaboratively by staff members from the UDRHs under the Student Survey Working Group (SSWG) of the Australian Rural Health Education Network (ARHEN). The survey tool contained quantitative and qualitative questions. Quantitative questions obtained information for participant characteristics and placement characteristics. Participant characteristics included age, gender, indigenous background ("Aboriginal," "Torres Strait Islander," "Both Aboriginal and Torres Strait Islander," "Neither Aboriginal nor Torres Strait Islander"), discipline, the name of the educational institute, and year of study. Placement characteristic included placement type, length, geographical location, and if interprofessional learning was 
provided ("Yes," "No," "Unsure"). Placement location was categorized utilizing the Australian Standard Geographical Classification-Remoteness Areas (ASGC-RA) classification system, ie, major city, inner regional area or large town (25,000-100,000), small rural town (10,000-24,999), small rural or remote community $(<10,000)$, or very remote center/ area. $^{14}$

Students' perception of the support received during their rural placements and changes in rural practice intentions was assessed through the following seven 5-point Likert scale statements ("strongly disagree" to "strongly agree"):

\section{Placement support and experience}

- "Received adequate indigenous cultural training during my placement."

- "Satisfied with the workplace supervision provided on my placement."

- "Received adequate educational resources during my placement."

- "Satisfied with my placement accommodation."

- "Overall, I was satisfied with my placement."

\section{Changes in rural practice intentions}

- "Prior to this placement, I was considering living and working in a regional, rural, or remote location following graduation."

- "This placement has made me reconsider my future, and I am now considering living and working in a regional, rural, or remote location following my graduation."

Qualitative data analysis addresses the following questions:

- What the students reportedly enjoyed most about their placement?

- What the experience was like of living and working in their placement location?

- How could their experience have been improved?

\section{Data analysis}

Descriptive and inferential statistics were used to summarize the quantitative data. Chi-square $\left(\chi^{2}\right)$ tests were performed to examine if there were any associations between variables. SDs and CIs are shown, where appropriate. Data were analyzed regarding student- and placement-level variables, eg, age, sex, indigenous status, placement location, duration, satisfaction with rural placement experience, and future intentions for rural practice.

Modified Poisson regression analysis with a robust error variance was performed to determine the relationship between placement satisfaction and future rural practice intentions. ${ }^{15}$ For regression analysis, Likert scale responses were reduced into dichotomous categories, representing the participant's choice between "disagreement" (strongly disagree/disagree) or "agreement" (strongly agree/agree). Significance was determined at two-tailed $P \leq 0.05$. All quantitative analyses were conducted using Stata v13.1.

Responses to an open-ended question about the graduates' perceptions of the effect of their rural placement were analyzed for themes. All responses were reviewed by three investigators (YF, SKing, SS) to identify patterns. All themes and subthemes were reviewed until no further themes could be identified, and the resulting themes accounted for all relevant information. Excerpts from survey responses are provided in the Results section to support further the authenticity of the themes derived.

\section{Results}

A total of 873 students who were enrolled in a health professional program at an Australian university and completed clinical placement at Australian rural and remote locations completed the evaluation survey during the academic years 2014-2016. In 2014, 2015, 2016, and 2017 surveys, 187, 202, 229 , and 255 students participated in the survey, respectively.

The majority of the survey respondents were females $(70.06 \%)$, with a median age of 22 years (IQR $21-25$ years). Only $2.65 \%$ of students identified themselves as indigenous Australians. Around half of the survey respondents were medical students $(44.46 \%)$, with the least representation $(2.42 \%)$ from dentistry students. Students were enrolled at 11 Australian universities. For nearly half of the participants (47.11\%), placement duration was $>4$ weeks to $\leq 3$ months. There was considerable variation in placement duration across different disciplines. Compared with nursing (29\%) and pharmacy (10\%) students, significantly higher percentage of medicine $(67 \%)$ and allied health students (100\%) spent more than 1 month on rural/remote placements. A significant proportion, ie, $76 \%$ of students, reported being at remote locations in their placement period (ASGC-RA), while the rest reported their placement locations at very remote locations. The profile of student demographics, academic discipline, and placement-related variables is presented in Table 1.

Overall, students reported high levels of satisfaction (93.93\%) with their placement experience. Of all constituents of placement experience, the highest level of satisfaction was reported for placement accommodation (91.44\%). Students also reported a high level of satisfaction with workplace 
Table I Attributes of students on clinical placement at MICRRH between January 2014 and December 2016, based on student placement survey conducted by MICRRH, Queensland, Australia

\begin{tabular}{|c|c|}
\hline Demographics & $\mathbf{N}(\%)$ \\
\hline \multicolumn{2}{|l|}{ Gender } \\
\hline Female & $433(70.52)$ \\
\hline Male & I8I (29.48) \\
\hline \multicolumn{2}{|l|}{ Indigenous status } \\
\hline Nonindigenous & $598(97.08)$ \\
\hline Indigenous & $18(2.92)$ \\
\hline Median age (IQR) & 22 years ( $2 \mathrm{I}-24$ years) \\
\hline \multicolumn{2}{|l|}{ Discipline } \\
\hline Medicine & $291(47.16)$ \\
\hline Nursing & 77 (I2.48) \\
\hline Pharmacy & $113(18.31)$ \\
\hline Allied health & $114(18.48)$ \\
\hline Others & $22(3.57)$ \\
\hline \multicolumn{2}{|l|}{ Year of study } \\
\hline First to third year & $211(34.20)$ \\
\hline Fourth year & $253(41.00)$ \\
\hline Fifth to sixth year & $130(21.07)$ \\
\hline Postgraduate & $23(3.73)$ \\
\hline \multicolumn{2}{|l|}{ University } \\
\hline James Cook University & $403(65.42)$ \\
\hline Rest of the Queensland & $104(16.88)$ \\
\hline Interstate & $109(17.69)$ \\
\hline \multicolumn{2}{|l|}{ Placement length } \\
\hline$>$ I week to $\leq 2$ weeks & $94(15.26)$ \\
\hline$>2$ weeks to $\leq 4$ weeks & $198(32.14)$ \\
\hline$>$ I month to $\leq 3$ months & $277(44.97)$ \\
\hline$>3$ months & $47(7.63)$ \\
\hline \multicolumn{2}{|l|}{ Placement type } \\
\hline Public hospital & $442(72.22)$ \\
\hline Community pharmacy & $54(8.74)$ \\
\hline Community health center & $45(7.28)$ \\
\hline Others & $71(11.60)$ \\
\hline \multicolumn{2}{|l|}{ Placement location } \\
\hline Remote & $482(77.99)$ \\
\hline Very remote & $133(21.52)$ \\
\hline
\end{tabular}

Abbreviation: MICRRH, Mount Isa Centre for Rural and Remote Health. supervision (89.87\%), educational resources $(87.75 \%)$, and indigenous cultural training $(90.87 \%)$ with interprofessional learning experience $(78.19 \%)$ provided during the placement (Table 2).

Postplacement intention to enter rural practice was strongly associated with overall placement satisfaction, as students who were happy with their placement experience reported higher rates for future rural/remote practice intentions than the students who were not satisfied with their placement experience ( $83.40 \%$ vs $63.64 \%, P<0.051$; chi-squared). Overall, postplacement intention for rural/remote practice was significantly higher from preplacement intentions. For those who initially were not interested in future rural/remote practice, nearly $75 \%$ of them changed their preference and reported positive intentions for future rural/remote practice postplacement.

Regression analyses indicate that in the adjusted models there was not any significant role of discipline, age, gender, and indigeneity. Students who were satisfied with their placement were significantly more likely (rate ratio [RR]: 2.10, 95\% CI: $1.03-4.26)$ to have rural practice intention than their counterparts. Of all components of rural placement experience, workplace supervision had the highest impact on students' intentions for future rural/remote practice (RR: 1.70, 95\% CI: 1.06-2.70). Placement length also seemed to play some role as students who stayed for $\geq 2$ weeks to $<4$ weeks were significantly more likely to have rural/remote practice intentions than students on $<2$ weeks of placement duration (RR: 1.08, 95\% CI: 1.00-1.17). Students who were happy with placement experience were significantly more likely (RR: 2.13, 95\% CI: 1.05-5.12) than their counterparts to change their future rural/remote practice intentions from negative to positive (Table 3).

Table 2 Health care students' views on placement experience offered by remote clinical placement site (Mount Isa Centre for Rural and Remote Health, based on student placement survey conducted by Mount Isa Centre for Rural and Remote Health, Queensland, Australia)

\begin{tabular}{|c|c|c|c|}
\hline \multirow[t]{2}{*}{ Placement experience } & \multicolumn{3}{|l|}{ Response, N (\%) } \\
\hline & $\begin{array}{l}\text { Strongly } \\
\text { disagree/disagree }\end{array}$ & Neutral & $\begin{array}{l}\text { Agreel } \\
\text { strongly agree }\end{array}$ \\
\hline Placement provided interprofessional learning experience/s & $39(6.33)$ & $43(6.98)$ & $534(86.69)$ \\
\hline Received adequate indigenous cultural training during my placement & $26(4.24)$ & $53(8.65)$ & $534(87.11)$ \\
\hline I was satisfied with the workplace supervision provided on my placement & $26(4.23)$ & $40(6.50)$ & $549(89.27)$ \\
\hline I received adequate educational resources during my placement & $28(4.57)$ & 49 (7.99) & $536(87.44)$ \\
\hline I was satisfied with my placement accommodation & $8(1.31)$ & $42(6.89)$ & $560(91.80)$ \\
\hline Overall, I was satisfied with my placement & II (I.79) & $27(4.40)$ & $575(93.80)$ \\
\hline
\end{tabular}


Table 3 Factors associated with future rural/remote practice intentions based on student placement survey (2014-2016), conducted by Mount Isa Centre for Rural and Remote Health, Queensland, Australia

\begin{tabular}{|c|c|c|}
\hline Future rural/remote practice intentions & Adjusted* rate ratio & $95 \% \mathrm{Cl}$ \\
\hline \multicolumn{3}{|l|}{ Model I } \\
\hline Satisfaction with accommodation (Yes) & 1.40 & $1.06-1.86$ \\
\hline Satisfaction with supervision (Yes) & 1.41 & $1.05-1.9 \mid$ \\
\hline Preplacement intention for rural practice (Yes) & 1.34 & $1.17-1.53$ \\
\hline Satisfaction with placement experience (Yes) & 1.80 & $1.06-3.07$ \\
\hline \multicolumn{3}{|l|}{ Changes in future rural/remote practice intentions } \\
\hline \multicolumn{3}{|l|}{ Model 2} \\
\hline \multicolumn{3}{|l|}{ "No" to "Yes" } \\
\hline Satisfaction with accommodation (Yes) & 4.47 & $1.22-16.40$ \\
\hline \multicolumn{3}{|l|}{ Model 3} \\
\hline \multicolumn{3}{|l|}{ "Yes" to "No" } \\
\hline Overall satisfaction with the placement experience (Yes) & 2.47 & $1.46-4.17$ \\
\hline
\end{tabular}

Notes: "Yes" includes "Strongly agree" and "Agree" responses, "No" includes "Strongly disagree" and "Disagree" responses. *Adjusted for age, gender, indigeneity, discipline, year of study, placement duration, and remoteness.

\section{Qualitative analysis}

The qualitative analysis led to the emergence of the themes related to positive and negative experience and suggestions for improvement. The following themes emerged from qualitative data analysis:

\section{A wide variety of experience and hands-on learning opportunities}

Students highlighted that working in remote location provides a unique learning experience as there is exposure to a wide variety of cases and models of service delivery. Students across the disciplines shared similar feelings and reflections:

An expanded scope of practice and responsibility, being able to utilise practical skills and apply knowledge learned in the course of my degree in a professional practice setting.

The wide scope of practice provided opportunity to experience a range of occupational therapy roles, professional development opportunities in specific areas, linking in with other disciplines and health services with the new graduate OT.

Students also highlighted that working in remote communities enables them to use skills learned in the clinical school and confers a higher sense of autonomy and responsibility.

It was eye-opening working with so many people and using skills I'd only known in theory before.

\section{Multidisciplinary exposure at home and workplace}

This was one of the recurring themes emerged from the qualitative analysis where students described the experience to be a part of a multidisciplinary team as an important learning experience. As highlighted in the following example:
I enjoyed having the opportunity to be placed with allied health professionals. It broadened my understanding of how allied health services and pharmacy interplayed in primary healthcare.

Inter-professional learning sessions (IPLs) with other undergraduate students (eg, medical and psychology students). It was invaluable to do role plays with them to see their case-based problem solving and roles that differ from ours.

Student perceived that working in multidisciplinary team facilitated clinical knowledge acquisition and enhanced problem-solving skills. Participants identified that openness to share knowledge was important factors in having a good learning experience. Students perceived that sharing the accommodation with students from different disciplines gave them a chance to know more about the scope and value of other health care disciplines.

Living with other disciplines was a great way to foster interdisciplinary understanding working at community rehab and in different settings really helped me to expand my view of a speech pathologists' role.

Placing students together from different disciplines helped me see the different perspectives on the many aspects of health care that are involved in treating a patient.

\section{Support from the local UDRH}

Participants spoke of being welcomed to the placement by the UDRH staff and being introduced to the community as important factors in feeling comfortable with the new environment. Participants appreciated the effort of UDRH to provide them nice accommodation and familiarizing them with nuances of the community. 
Both preceptors were great! Very friendly and helpful. The student accommodation was also really good. I was provided with heaps of information on Longreach and things to do, as well as being given a really good orientation.

The accommodation was of the very high standard; I was impressed with the facilities immensely. Additionally, living with the other students and having that contact with people in other health courses was great.

Living conditions were really great, and everything was provided. I felt safe, and it was a convenient location to and from placement.

\section{Experiencing challenges of rural health care services} Students found that by living in these communities, they were able to get a first-hand experience of living in the remote communities that allowed them to increase their understanding and knowledge of rural/remote health care services.

Over my placement, I was exposed to many different communities from rural to very remote. It was very interesting to meet the people living there and see how they lived and also their outlook on healthcare.

I learned a lot about rural pharmacy practice and both the benefits and challenges of living and working in rural and remote places.

Some participants talked about how the experience had helped them learn the challenges of working in a remote location, and they become more appreciative of the efforts of rural health care providers.

The opportunity to visit remote sites and physically see the health issues that are in those areas and also see how the healthcare system is run at these remote sites.

\section{Learning of indigenous culture}

Students believed that remote placement provided them with an opportunity to learn about the indigenous culture and gain an understanding of cultural safety. As some participants commented:

It was a new experience to work in a community with a high Indigenous population and the considerations you have to remember in order to achieve good communication with the person you were talking to. The utmost important thing to remember is to be culturally aware and sensitive.

Participants identified that remote placement experience had their increased understanding of the complexity of indigenous health care in a primary health care context and helped them become more empathic in their professional lives.
Talking to aboriginal patients and participating in their community activity (NAIDOC week) has improved my understanding on their psychosocial issues.

I am passionate about Indigenous wellbeing, and this placement has demonstrated to me that they are not a community burdened by history, but instead they are a community that is resilient and culturally strong in the face of trauma and disadvantage.

\section{Minor themes}

Social isolation

Participants perceived that social isolation can be one of the hardest parts of remote or rural practice to the extent of feeling ignored during the clinical placement. Students suggested that connecting with the community helps in overcoming the "social isolation" during placement.

I think it is very important that you get involved in the community if you want this placement to be enjoyable, if you stay at home it is just going to seem like forever that you are on this placement.

Slightly isolating but built resilience and adaptability to finding new friends and community.

\section{Need for longer placements}

Some participants echoed the shorter duration of placement as an obstacle to achieve the learning objectives. This is illuminated by the following extract:

I noticed the three-week placement group had many more opportunities and I think it would be improved if the twoweek group was given these opportunities as well.

They mentioned that longer placement enhances their experience of living in a remote community and provides them with an opportunity to improve clinical skills and develop social and professional networks.

It would be convenient to stay in the same in the locality for the three weeks, fully immerse yourself into the community.

Three months is a considerable amount of time, and it really allows you to immerse yourself in the community.

\section{Discussion}

Our results provide conclusive evidence for a strong association between positive rural placement experience and future rural practice intentions. The findings of this survey indicate that students perceived that rural/remote placements helped them develop competencies, enhance their confidence, and introduce them to remote rural health care services and 
indigenous culture. Although social isolation on placement was considered as a negative experience, nonetheless support from the UDRH staff was widely appreciated. It is necessary to document that some students highlighted the need for extended placements to enhance their learning experience and preferred local placement for rural students. Overall, our results add to the literature that not only medical student but students from another discipline also look forward to employment in areas associated with positive placement experience.

This study also supports previous literature that found that hands-on learning and multidisciplinary exposure is an essential feature of rural placement experience. ${ }^{9,16}$ Students appreciate the multidisciplinary living exposure. Due to the staff shortage, health professionals working in rural/remote areas are required to work more generalist, collaboratively, and are more interdependent than those in more urban areas. ${ }^{17}$ Remote placement sites immerse students in problem-rich environments that introduce them to the challenges faced by both staff and patients in rural areas, enhance problemsolving, and facilitate learning by socialization with clients, community members, and other health professionals, ${ }^{18}$ thus turning up a potential deficit into a potential strategy for future workforce development.

One of the strategies that worked for maximizing the multidisciplinary exposure opportunities and building Collegian Network was to place students from different disciplines in the same accommodation deliberately, thus broadening their experience and providing them opportunities to build an interprofessional network, which is essential for practicing in remote and rural locations.

The expectations from clinical placement sites are to provide conducive environments that support and facilitate learning of placement students, which in turn may help in overcoming health workforce maldistribution over time. Rural placements need to be of sufficient duration to allow the prospective employee to experience the rural lifestyle and challenges of working in a resource-scarce setting, as well as providing an opportunity for potential employers to assess the potential of the future graduate employee. ${ }^{19}$ Our findings also highlight that placement duration is also a vital influencer of postplacement rural and remote practice intentions, as longer placement provide adequate time to immerse in the community and experience the rural lifestyle. Nonetheless, the positive thing is that the number of students coming on shorter placements is diminishing.

For students who were born and bred in metro cities and did not get an opportunity to interact with indigenous
Australians, rural/remote placements provide a valuable opportunity for connecting with remote-dwelling indigenous communities, understanding their connection with the land, and respecting their cultural heritage. Understandably, social interaction is an essential component of rural placement experience to learn about the beliefs, practices, and health issues of the community. The support and mentoring from local indigenous cultural mentor, placement site staff, and local health care providers are vital in ensuring that these future health care providers acquire confidence and skills to interact, collaborate with indigenous Australians, and enrich their placement experience.

Rural placement model needs to focus on socializing health care students into rural and remote communities to overcome the contextual barriers, eg, geographic isolation, family and relationship needs. ${ }^{20}$ The need for students to be connected virtually with their family friends and the home university could not be underemphasized. Therefore, access to the Internet is vital. It introduces the concept of telehealth to students and prepares a workforce who has experience in managing the challenges of geographic barriers in providing care.

Addressing student anxieties, needs, and continued efforts toward students' acclimatization in the community to help them become a part of the community could help in further engaging the students. Perhaps, student placement coordinator can help in overcoming this problem by providing orientation to the community, health issues of local community and important community events, as well as introducing them to residents.

Overall, this study demonstrates that positive rural placement experience plays a significant role in preparing future rural health workforce by engaging students on many levels, improving their understanding of rural communities, and providing a first-hand experience of rural health services issues. ${ }^{21}$ Nevertheless, there are a few caveats that are important to discuss. Because this was a repeated cross-sectional study, we relied on students' perceptions and recall of factors that influenced their recruitment decisions. Therefore, this study has limitations in assessing if these intentions translated into actual practice. A prospective study that surveys when initially recruited and following up later would provide a more accurate indication of the role of rural placement on future rural/remote practice. Strengths of this research include representation from multiple disciplines across Australia. Specifically, this study has added to previous findings by exploring the experience of pharmacy and allied health that are relatively unexplored in the existing literature. 


\section{Conclusion}

Positive placement experience does not constitute just one or two components but a multifaceted arrangement of different components that are developed considering students' professional and personal needs and overcoming geographic barriers. Our results provide evidence that facilitation of positive placement experiences in remote and rural locations could be a key strategy in addressing rural health workforce maldistribution. In the meantime, more research is needed to provide the data needed for the careful design of coursework, detailed planning of the placement experience, and long-term strategies to address negative aspects of rural/remote placement experience.

\section{Acknowledgments}

We acknowledge students who generously gave their time to participate in this study and ARHEN staff for their efforts in developing the survey tool. In particular, we would like to acknowledge Student Placement Officers who actively encouraged students to participate in this survey.

\section{Disclosure}

All authors declare that there are no conflicts of interest regarding the publication of this article.

\section{References}

1. Kondalsamy-Chennakesavan S, Eley DS, Ranmuthugala G, et al. Determinants of rural practice: positive interaction between rural background and rural undergraduate training. Med J Aust. 2015;202(1):41-45.

2. McAuliffe T, Barnett F. Factors influencing occupational therapy students' perceptions of rural and remote practice. Rural Remote Health. 2009;9(1):1078.

3. Walker JH, Dewitt DE, Pallant JF, Cunningham CE. Rural origin plus a rural clinical school placement is a significant predictor of medical students' intentions to practice rurally: a multi-university study. Rural Remote Health. 2012;12:1908.

4. Playford DE, Evans SF, Atkinson DN, Auret KA, Riley GJ. Impact of the Rural Clinical School of Western Australia on work location of medical graduates. Med J Aust. 2014;200(2):104-107.
5. King KR, Purcell RA, Quinn SJ, Schoo AM, Walters LK. Supports for medical students during rural clinical placements: factors associated with intention to practise in rural locations. Rural Remote Health. 2016;16(2):3791.

6. Tolhurst HM, Adams J, Stewart SM. An exploration of when urban background medical students become interested in rural practice. Rural Remote Health. 2006;6(1):452.

7. Sen Gupta TK, Muray RB, McDonell A, Murphy B, Underhill AD. Rural internships for final year students: clinical experience, education and workforce. Rural Remote Health. 2008;8(1):827.

8. Ranmuthugala G, Humphreys J, Solarsh B, et al. Where is the evidence that rural exposure increases uptake of rural medical practice? Aust J Rural Health. 2007;15(5):285-288.

9. Orpin P, Gabriel M. Recruiting undergraduates to rural practice: what the students can tell us. Rural Remote Health. 2005;5(4):412.

10. Crowe MJ, Mackenzie L. The influence of fieldwork on the preferred future practice areas of final year occupational therapy students. Aust Occup Ther J. 2002;49(1):25-36.

11. Wilkinson D, Birks J, Davies L, Margolis S, Baker P. Preliminary evidence from Queensland that rural clinical schools have a positive impact on rural intern choices. Rural Remote Health. 2004;4(4):340.

12. Sen Gupta T, Murray R, Hays R, Woolley T. James Cook University MBBS graduate intentions and intern destinations: a comparative study with other Queensland and Australian medical schools. Rural Remote Health. 2013;13(2):2313.

13. Neill J, Taylor K. Undergraduate nursing students' clinical experiences in rural and remote areas: recruitment implications. Aust J Rural Health. 2002;10(5):239-243.

14. McGrail MR, Humphreys JS. Geographical classifications to guide rural health policy in Australia. Aust New Zealand Health Policy. 2009;6:28.

15. Zou G. A modified Poisson regression approach to prospective studies with binary data. Am J Epidemiol. 2004;159(7):702-706.

16. Gum LF, Richards JN, Walters L, Forgan J, Lopriore M, Nobes C. Immersing undergraduates into an interprofessional longitudinal rural placement. Rural Remote Health. 2013;13(1):2271.

17. Kelley ML, Maclean MJ. Interdisciplinary continuing education in a rural and remote area: the approach of the northern educational centre for aging and health. Educ Gerontol. 1997;23(7):631-649.

18. Penman J, Oliver M. Meeting the challenges of assessing clinical placement venues in a bachelor of nursing program. J Univ Teach Learn Pract. 2004;1(2):59-73.

19. Wilkinson D, Laven G, Pratt N, Beilby J. Impact of undergraduate and postgraduate rural training, and medical school entry criteria on rural practice among Australian general practitioners: national study of 2414 doctors. Med Educ. 2003;37(9):809-814.

20. Roberts C, Daly M, Kumar K, Perkins D, Richards D, Garne D. A longitudinal integrated placement and medical students' intentions to practise rurally. Med Educ. 2012;46(2):179-191.

21. Webster S, Lopez V, Allnut J, Clague L, Jones D, Bennett P. Undergraduate nursing students' experiences in a rural clinical placement. Aust J Rural Health. 2010;18(5):194-198.
Journal of Multidisciplinary Healthcare

\section{Publish your work in this journal}

The Journal of Multidisciplinary Healthcare is an international, peerreviewed open-access journal that aims to represent and publish research in healthcare areas delivered by practitioners of different disciplines. This includes studies and reviews conducted by multidisciplinary teams as well as research which evaluates the results or conduct of such teams or health

\section{Dovepress}

care processes in general. The journal covers a very wide range of areas and welcomes submissions from practitioners at all levels, from all over the world. The manuscript management system is completely online and includes a very quick and fair peer-review system. Visit http://www.dovepress.com/ testimonials.php to read real quotes from published authors. 